\title{
Characterization of surface forces for electro-mechanical memory cells
}

\author{
Woo Young Choi ${ }^{\text {a) }}$ \\ Department of Electronic Engineering, Sogang University 1 Shinsu-dong, Mapo-gu, \\ Seoul 121-742, Republic of Korea \\ a)wchoi@sogang.ac.kr
}

\begin{abstract}
By comparing experimental and simulation data, surface forces of electro-mechanical memory cells were characterized. In the case of aluminum beams, surface forces were negligible due to rough surface topology. However, in the case of titanium-nitride beams, surface force density was estimated to be $624 \mathrm{kPa}$. The extracted value of surface force can be fed back into the finite-element-analysis (FEA) simulation for better modeling accuracy.
\end{abstract}

Keywords: surface force, electro-mechanical memory cell

Classification: Electron devices, circuits, and systems

\section{References}

[1] W. Y. Choi, H. Kam, D. Lee, J. Lai, and T.-J. King Liu, "Compact nanoelectro-mechanical non-volatile memory (NEMory) for 3D integration," Tech. Dig. International Electron Devices Meeting (IEDM), Washington, DC, USA, pp. 603-606, Dec. 2007.

[2] C. H. Mastrangelo and C. H. Hsu, "A simple experimental technique for the measurement of the work of adhesion of microstructures," Proc. IEEE Solid State Sensors and Actuators Workshop, Hilton Head Island, SC, USA, pp. 208-212, June 1992.

[3] M. R. Houston, R. Maboudian, and R. T. Howe, "Ammonium fluoride anti-stiction treatments for polysilicon microstructures," Proc. 8th Int. Conf. Solid-State Sensors and Actuators-Transducers '95, Stockholm, Sweden, pp. 210-214, June 1995.

[4] M. R. Houston, R. T. Howe, and R. Maboudian, "Effect of hydrogen termination on the work of adhesion between rough polycrystalline silicon surfaces," J. Appl. Phys., vol. 81, no. 8, pp. 3474-3483, April 1997.

\section{Introduction}

As an alternative to conventional flash memory technology, electro-mechanical memory cells have attracted much attention. Previously, a novel electromechanical memory cell has been proposed for nonvolatile memory applications as shown in Fig. 1 (a) [1]. The proposed electro-mechanical memory cells generally have small volume, large surface area, and narrow air gap, 
so that surface forces can increasingly affect performance and reliability as the cell size is scaled down. Thus, the characterization of surface forces between the bit line and the oxide/nitride/oxide (ONO) layer is indispensable to provide guidelines for electro-mechanical memory cell design.

Many papers have been published for simple and accurate characterization of surface forces. The most widely used method is to fabricate an array of cantilevers of different lengths on the same die [2, 3, 4]. Adhesion can be forced by applying pressure from a probe. After the pressure is removed, the array is examined to find the shortest cantilever beam remaining in contact with the substrate. From the length of this beam, the surface adhesion energy can be determined. However, sticking of the cantilever beam to the probe tip which is used to apply pressure is a problem for this method. In order to address this issue, a modified version of the method utilized electrostatic force instead of pressure from a probe.

However, these two methods are difficult to use for characterization of surface forces of the electro-mechanical memory cells shown in Fig. 1 (a). In the proposed electro-mechanical memory cell, the bit line is covered by the read word line and passivation layer, which means the movement of the bit line cannot be observed by using microscopes. Also, the thickness of air gap is too small to observe the change in cantilever beam profile and determine contact area sizes. Thus, in this study, we used an indirect method to determine surface forces and energy: pull-in and release voltages are measured experimentally and compared against simulated values in order to estimate the surface forces.

\section{Surface force characterization}

In order to quantify surface forces, we fabricated cantilever-type electromechanical memory cells using two kinds of beam material: aluminum and titanium nitride as shown in Fig. 1 (a). Fig. 1 (b) and 1 (c) show the scanning electron microscopy (SEM) images of the fabricated cells. Table I summarizes the design parameters of each cell. The two memory cells have the same design parameters except for beam length $\left(L_{\text {beam }}\right)$ and width $\left(W_{\text {beam }}\right)$. To determine surface forces, finite-element analysis (FEA) was performed. In FEA simulation, surface forces were quantified by a two-step process: pull-in voltage $\left(V_{\text {pull-in }}\right)$ and release voltage $\left(V_{\text {release }}\right)$ modeling. In the first step, $V_{\text {pull-in }}$ modeling step is necessary for calibration: the Young's modulus of the cantilever beam used for the FEA is adjusted so that the simulated $V_{\text {pull-in }}$ equals the measured $V_{\text {pull-in }}$. In the second step, $V_{\text {release }}$ modeling is done. Since surface forces are not considered in the FEA simulation, the difference of hysteresis voltage $\left(V_{\text {pull-in }}-V_{\text {release }}\right)$ between the FEA-simulated and measured data can be attributed to surface forces. Fig. 2 (a) shows the aluminum beam case. Since there is no difference between the measured and simulated $V_{\text {release }}$ 's, it can be inferred that the surface forces are negligible. However, for the case of a titanium nitride beam shown in Fig. 2 (b), 0.2-V difference between the measured and simulated $V_{\text {release's is observed, indicating }}$ 


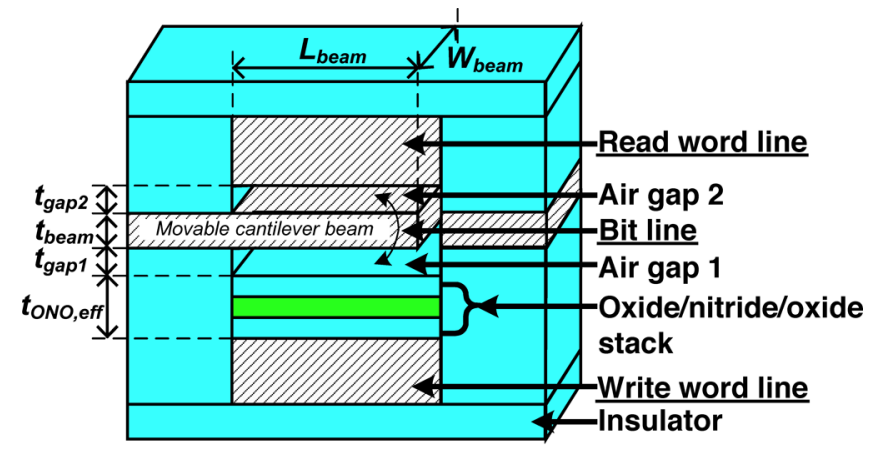

(a)

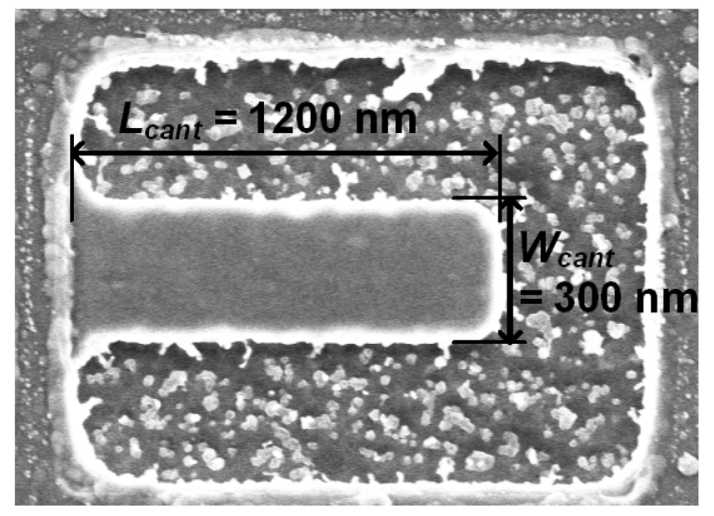

(b)

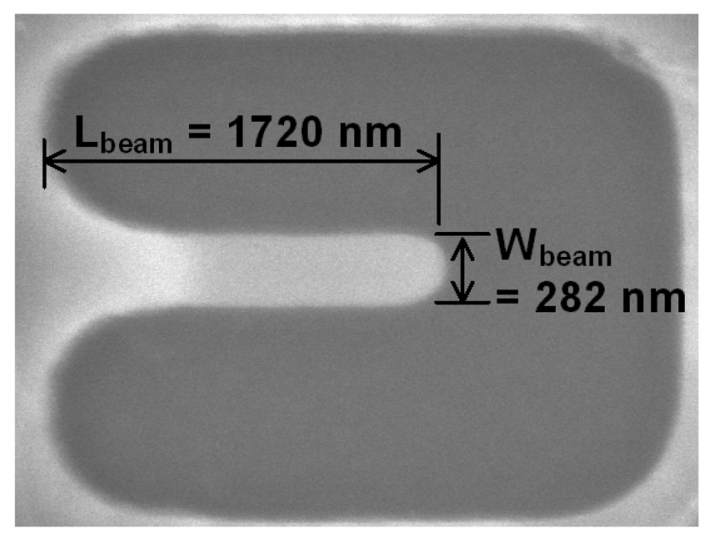

(c)

Fig. 1. (a) Schematic of cantilever-type electromechanical memory cell. (b) SEM image of fabricated aluminum electro-mechanical memory cell. (c) SEM image of fabricated titanium nitride electro-mechanical memory cell.

that surface forces influence cell characteristics. The surface force difference between the aluminum and titanium nitride cases stems from a difference in surface roughness: generally, aluminum beams have a much rougher surface than titanium beams due to spiking of aluminum into the sacrificial amorphous silicon material; as a result, contact between an aluminum bit line and the ONO surface is made only at some asperities, resulting in negligible surface adhesion force. Thus, surface forces are characterized only in the titanium nitride beam case. 
Table I. Design parameters of the fabricated electromechanical memory cells shown

\begin{tabular}{|c|c|c|}
\hline Beam material & Aluminum & Titanium nitride \\
\hline Beam length $\left(L_{\text {beam }}\right)(\mathrm{nm})$ & 1200 & 1720 \\
\hline Beam width $\left(W_{\text {beam }}\right)(\mathrm{nm})$ & 300 & 282 \\
\hline Beam thickness $\left(t_{\text {beam }}\right)(\mathrm{nm})$ & 100 & 100 \\
\hline Air-gap thickness $\left(t_{\text {gap } 1,2}\right)(\mathrm{nm})$ & 30 & 30 \\
\hline ONO-layer thickness & $\begin{array}{c}6 \text { (oxide) } \\
6 \text { (nitride) } \\
3 \text { (oxide) }\end{array}$ & $\begin{array}{c}6 \text { (oxide) } \\
6 \text { (nitride) } \\
3 \text { (oxide) }\end{array}$ \\
\hline
\end{tabular}

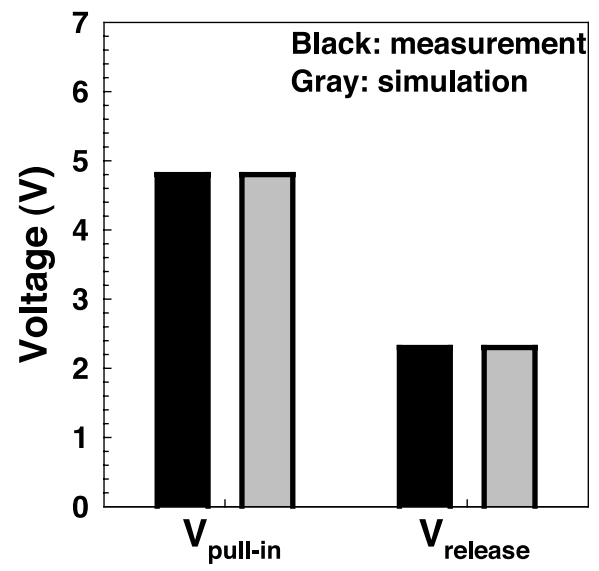

(a)

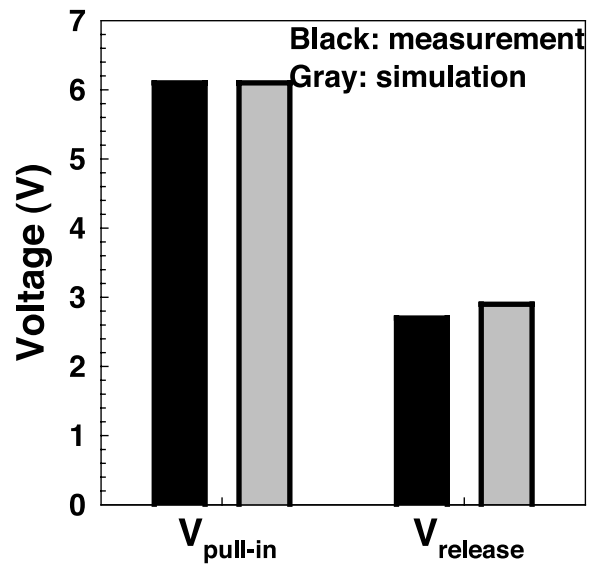

(b)

Fig. 2. Comparison of measured and FEA-simulated $V_{\text {pull-in's }}$ and $V_{\text {release's. (a) Aluminum electro- }}$ mechanical memory cell. (b) Titanium nitride electro-mechanical memory cell.

In order to extract surface forces of titanium nitride beams, in the second step, surface forces are included in the FEA simulation and their values are adjusted in order to obtain a match between the simulated and mea- 
sured values. The modeling results reveal that $30-\mathrm{nN}$ surface force exists over an apparent contact area of $48500 \mathrm{~nm}^{2}$ in the case of titanium nitride beams. Thus, the surface force density is estimated to be $624 \mathrm{kPa}$ which is not ignorable when modeling electro-mechanical memory cells.

\section{Conclusions}

Surface forces of electro-mechanical memory cells have been characterized by using the measured and FEA-simulated data. Because it is impossible to observe beam movement of electro-mechanical memory cells by using microscopes, an indirect method has been proposed. According to the characterization results, contrary to aluminum beams, titanium nitride beams show significant surface forces. The extracted values of surface forces will be helpful in improving the accuracy of electro-mechanical memory cell modeling.

\section{Acknowledgments}

This work was supported in part by the National Research Foundation of Korea, funded by the Ministry of Education, Science and Technology, under Grants 2009-0082439 and 2009-0084522 and in part by the Korea Sanhak Foundation under a 2009 research grant. 